\title{
Multimethodology in Metaheuristics
}

\author{
J Soeiro Ferreira ${ }^{1,2 *}$ \\ ${ }^{1}$ Faculdade de Engenharia da Universidade do Porto, Porto, Portugal; and ${ }^{2}$ INESC TEC-Instituto de \\ Engenharia de Sistemas e Computadores, Porto, Portugal
}

As a combination of different methodologies or parts of methodologies, Multimethodology is becoming more frequent in OR practice. This paper contributes with a new proposal and a new field of application: the employment of Multimethodology in problem solving with Metaheuristics (Mh). A convenient selection of soft and hard methods will be considered, from Soft OR, Creativity and Metaheuristics, such as Strategic Choice Approach, SWOT Analysis and Divergent and Convergent thinking. Formulating the 'right' optimisation problem, choosing a method based on $\mathrm{Mh}$ and accomplishing an effective implementation is an imprecise decision-making process, which may require skills and ideas that are beyond the ordinary boundaries of Mh practice. The relevance and success of Mh have been well-known for decades, but some open questions concerning choice and implementation strategies, for instance, still remain. If these questions are not adequately answered, they may lose credibility in the long term. The quality of solutions and computational times are not the only criteria used to analyse Mh, nor are they the most important. Very often, the effectiveness of an approach has to be evaluated from the perspective of modelling and practical problem solving. This paper investigates the advantages of Multimethodology and, furthermore, it sketches a framework for a coherent and comprehensive comparison of $\mathrm{Mh}$ and recommends a dynamic guiding tool for their implementation.

Journal of the Operational Research Society (2013) 64, 873-883. doi:10.1057/jors.2012.88

Published online 5 September 2012

Keywords: Multimethodology; soft OR; creativity; Metaheuristics; strategic choice approach; SWOT analysis

\section{Introduction}

Multimethodology may help to address different levels and dimensions of problem solving with Metaheuristics (Mh). Multimethodology is the employment and combination of several methods or parts of methods, both quantitative (hard) and qualitative (soft), together in a single engagement (Mingers, 2000). Mixing methods in different ways is a rapidly emerging investigation area to both OR researchers and practitioners, but also to researchers in other fields. In this paper, a plurality of ideas, methods and tools of Soft Operational Research (Soft OR), of Creativity and of $\mathrm{Mh}$ will be utilised in problem solving. $\mathrm{Mh}$ practice is not a straightforward endeavour, namely when dealing with real-life problems with technical, personal and social dimensions. The researchers commonly face various situational characteristics which are ambiguous, loosely defined and where 'objectivity' is not generally possible. The individual practitioner (or team), the interdependence of modelling and $\mathrm{Mh}$ choice, the computational complexity, Mh implementations and the evaluation of the solutions are all elements of this complex working setting. Even for a well-defined optimisation

\footnotetext{
*Correspondence: J Soeiro Ferreira, FEUP/INESC TEC, Campus da FEUP, Rua Roberto Frias, Porto 4200-465, Portugal.

E-mail: jose.soeiro.ferreira@inescporto.pt
}

problem, the 'simple' choice of a solution method and its effective implementation is an imprecise decisionmaking process. Thus it can be argued that the improvement of this 'wicked situation' requires skills and ideas beyond the ordinary thinking of the specific $\mathrm{Mh}$ area.

Mh's relevance, power and success have been wellknown for decades. However, open questions still remain: how to choose a Mh. In the presence of a concrete optimisation problem - which effective, efficient $\mathrm{Mh}$ (that is able to produce an 'optimal' solution), at the cost of a reasonable computing time, should be selected? And following this selection (how to do it?), there is no universal way of improving it, to elect adequate strategies. The choice of a good $\mathrm{Mh}$ and the adjustment of the correspondent parameters suggest, as mentioned, the adoption of innovative ideas and tools, in addition to the valuable expertise and intuition of the researchers. The quality of solutions and computational times are not the only criteria for analysing or choosing an $\mathrm{Mh}$, nor are they necessarily the most vital. The effectiveness of a solution approach should also be evaluated in the context of practical problem solving, particularly if the complexity of the problem is very high and the models are simplistic. Methods and solutions should not be isolated from the problematic of understanding the 'right' problem and from the agreement on a convenient (usually approximate) model. Flexibility, 
analysability, robustness, appeal, experience and developments in computer technology may represent other criteria that should be taken into account.

This paper investigates and explores Multimethodology from an experiential and a conceptual perspective, ranging from Soft OR methods, also known as Problem Structuring Methods (PSM), and Creative thinking to $\mathrm{Mh}$, to dealing with complex optimisation problems. The resulting body of work supports different stages of a project with $\mathrm{Mh}$ and, in particular, it sketches a framework for a coherent and comprehensive evaluation of dissimilar approaches based on Mh. This is an innovative challenge, especially due to the types of methods combined, soft and hard that originate from different areas.

The outline of the paper is as follows. After presenting the issues and proposals of the work, the next section introduces Soft methods/PSM, it outlines Creative Problem Solving (CPS) and introduces Mh. The objective is to relate and accommodate the different themes, in the context of Multimethodology in Mh practice. Section 3 is dedicated to choosing $\mathrm{Mh}$ and, in particular, the use of SWOT Analysis is addressed. Tools from the Strategic Choice Approach (SCA) are proposed in Section 4, to deal with a comprehensive comparison and evaluation of $\mathrm{Mh}$. A case of comparing methods used to solve an Arc Routing Problem is also included in order to illustrate Multimethodology. Section 5 brings a dynamic structure, supported by CPS phases of Divergent and Convergent thinking, to guide and facilitate the process of implementing Mh. Some conclusions are drawn in Section 6. Finally, a list of references is included.

\section{Multimethodology and Metaheuristics}

As mentioned in Kotiadis and Mingers (2006) ' ... significant benefits can accrue by combining different methodologies together-what has been termed Multimethodology. This can consist of simply combining several PSM together, which is relatively unproblematic, or it can involve combining PSM with more traditional hard techniques'. A variety of approaches have been undertaken when mixing methods, which are mentioned in generic references to Multimethodology such as Eden et al (2009), Howick and Ackermann (2011), Mingers and Brocklesby (1997), Mingers and Gill (1997), Mingers (2001), Munro and Mingers (2002) and Ormerod (2001). These works also review published case studies where different methods have been mixed and applied to various interventions, or share the lessons gained from practical experiences or suggest opportunities for further research. Empirical evidence shows that combining PSM/Soft OR with hard techniques has occurred less often (Munro and Mingers, 2002). The following works are some examples of mixing soft and hard methods. In Bell et al (2009) the holon framework is developed, as a Multimethodology merging both soft and hard issues. Aspects of Soft Systems Methodology (SSM) are used to address the soft part of the framework and metrics are collected through the use of the Goal/Question/Metric method; the paper (Mingers et al, 2009) reports a study evaluating the performance of the basic research institutes of the Chinese Academy of Sciences, through an innovative combination of SSM with data envelopment analysis (DEA). SSM is specially used in defining and agreeing appropriate inputs and outputs for DEA, which led to a comprehensive and systemic database of performance indicators; the paper (Pollack, 2009) presents two distinct ways in which hard and soft methods can be combined: in series and in parallel. Accordingly, Multimethodology in parallel, less common, has the potential to provide significant benefits to projects in political, changing, or 'wicked' contexts that in series cannot. SSM and Project Management were combined in a project in the Australian public sector; the authors of Mabin et al (2009) suggest the use of the Evaporating Cloud (EC) approach in multimethodological interventions. In particular they report the improvements of the benefits of Mixed Integer Programming by initially using the EC approach in the facilities location problem. Mixing methods is common in other areas, such as sociology, anthropology and environment (Driscoll et al, 2007; Johnson and Onwuegbuzie, 2004; Teddlie and Tashakkori, 2009).

There are advantages and a decisive value in resorting to Multimethodology to deal with complex optimisation problems, and this paper deliberately aims to contribute at this level by mixing and combining soft and hard approaches, in particular those that range from Soft OR, Creativity and Mh. Real-life problems do not arrive on decision makers' desks with a clear identification or label. Understanding and agreeing on the problem/model, discussing the approaches and implementing the solution methods should not be isolated. In particular, selecting an $\mathrm{Mh}$ for a particular application is not objective or 'rational', it may be a personal or group matter that is subject to motivations, familiarities and the working environment. Developing, implementing and comparing $\mathrm{Mh}$ are themselves problem-solving activities. There is a debate regarding the exact meaning of the terms 'methodology' and 'method'-this paper uses them indifferently. In this work, parts of methodologies are essentially brought together to support Mh practice.

\subsection{Soft OR methods}

Soft methods/PSM have been being developed over the last 40 years. The emphases of these methods are: structuring messy, complex situations that are full of uncertainties and lack agreed objectives, as opposed to solving well-defined problems; exploring the differing views of the participants; and facilitating participation and engagement, and analysing 
quantitative aspects and mathematical models. The intention is to deal with 'wicked problems' which seem to depart from the application of 'traditional' OR methods. Probably the most well-known are: Soft Systems Methodology (SSM), Strategic Choice Approach (SCA) and Strategic Options Development and Analysis (SODA). Generic references include Keys (2006), Pidd (1996) and Rosenhead and Mingers (2001). Related references, Multimethodology included, are: Kotiadis and Mingers (2006) and Mingers (2000). Joldersma and Roelofs (2004), Paucar-Caceres (2009), Vidal (Guest Editor) (2004), White (2009) and Winterfeldt and Fasolo (2009) also highlight the topic of Soft methods and their applications.

SCA (Friend and Hickling, 2005) is one of the methods that will be used in connection with $\mathrm{Mh}$ practice. The following characterisation of SCA is taken from Friend (2001): ' . . . deals with the interconnectedness of decisions problems in an explicit yet selective way. Its most distinctive feature is that it helps people working together to make more confident progress towards decisions by focusing their attention on possible ways of managing uncertainty as to what they should do next'. Three broad types of uncertainties are typified, each requiring a different style of response: uncertainties relating to the work environment, uncertainties relating to guiding values and uncertainties relating to choices on related agendas. In the following SCA working modes, these uncertainties may become apparent. SCA begins with a set of related decision problems and decision areas that are under consideration, and distinguishes four complementary modes: the Shaping mode, the Designing mode, the Comparing mode and the Choosing mode (Figure 1). These are briefly defined as follows:

- The Shaping mode: the decision-makers will consider the various decision areas in terms of their interrelationships and relative importance. The aim is to consider whether the current focus is appropriate or, conversely, it should be enlarged.

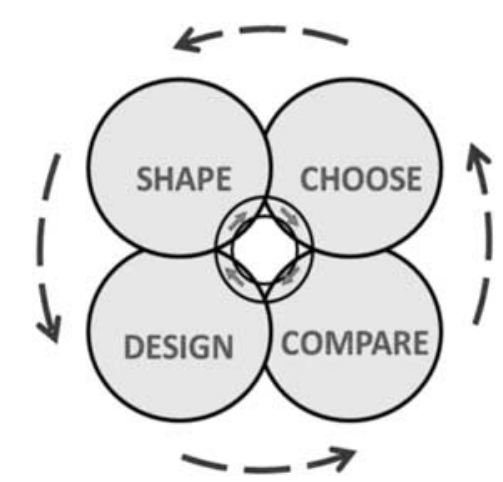

Figure 1 Four modes in a process of strategic choice.
- The Designing mode: the decision-makers will devise which schemes of action are feasible in relation to their current view of the problem. The options for each decision area are examined in pairs to test incompatibility. All of the possible combinations of options are then considered in order to arrive at the feasible decision schemes.

- The Comparing mode: when functioning in this mode, the feasible decision schemes are compared by evaluating them in terms of several comparison areas which have been identified by the participants. A pair-wise comparison of the decision schemes is undertaken using a comparative advantage grid. It is known that uncertainty of the different types mentioned earlier will become apparent in these modes.

- The Choosing mode: in this mode, the participants will look for an agreement and a commitment to action. Remaining uncertainties may delay some decisions until exploratory actions have occurred to reduce them. The agreed combination of commitments and future explorations may be expressed in a commitment package.

SWOT Analysis is a framework for evaluating the Strengths, Weaknesses, Opportunities and Threats involved in a project or activity that has been widely used, in particular in strategic planning (Weihrich, 1982; Panagiotou, 2003; Sodhi and Tang, 2008). It is a helpful structuring method, which may be related to Soft OR/ PSM, that facilitates the scanning of the internal and external factors as an important part of a strategic planning or decision process. At a practical level the analysis results in a SWOT (or TOWS) matrix/template which is a good tool to list and combine these factors. SWOT may also be tailored to study and analyse the advantages and drawbacks of resolution methods. In Section 3 SWOT will be applied in the new context of $\mathrm{Mh}$ and to complement the overview of a selected $\mathrm{Mh}$.

Soft methods may be utilised by individuals but they meet their full potential when used with groups. They often integrate phases of 'brainstorming', a term that probably originated with Osborn (1953), and facilitation practices in workshop sessions (Papamichail et al, 2007) in which a model or models representing the problem situation are built up and worked out, through the collaboration of a facilitator and the group involved. In this context, the facilitator will promote periods to generate and create many ideas (without evaluation) and periods to choose the best ideas, and to formulate and develop them on more concrete terms. These are also widespread procedures in Creativity and Creative Problem Solving (CPS) and they are known as the Divergent Thinking and Convergent Thinking phases. The benefits of combining methods are well documented in the literature (Mingers and Brocklesby, 1997; Mingers, 2000). 


\subsection{Creativity}

$\mathrm{Mh}$ are an amazing illustration of creativity. They represent powerful novel optimisation methods that result from unconventional thinking and combine different areas of knowledge.

The book The Art of Problem Solving (Ackoff, 1978) is probably the first book on creativity in OR. Other interesting papers by the same author are Ackoff and Vergara (1981) and Ackoff (1987), which also raise the question about the limitations of traditional modelling and the importance of new approaches. Herrmann (1996) gives a short definition of creativity that encapsulates many other definitions: 'Among other things, it is the ability to challenge assumptions, recognize patterns, see in new ways, make connections, take risks, and seize upon chance'. Multimethodology, combining soft and hard approaches in problem solving, may also find significance within the statement, taken from Rubinstein (1986): 'The most creative human problem solvers have an unusual capacity to integrate the two modes of conscious functions of the two brain hemispheres, and move back and forth between the holistic and sequential, between intuition and logic, between the fuzzy field of a problem domain and a clear specific small segment of a field. Such people can be outstanding artists and scientist because they combine the strong attributes of both'. Selected general references in creativity include Glass (2006), Runco (2007) and, in the context of problem solving and OR practice, Marakas and Elam (1997), Vidal (2004, 2006). There are several programs and multiple step methods to boost Creativity, such as Synetics (Gordon, 1961), CPS-Creative Problem Solving (Osborn, 1953; Parnes, 1997), Lateral Thinking (De Bono, 1992), Brainstorming (Osborn, 1953) and Divergent Thinking/Convergent Thinking (Guilford, 1967).

A prime component of Creativity appears to be Divergent thinking. During a creative process it is convenient to start with Divergent thinking to produce as many ideas or solutions as possible and thereafter to switch to Convergent thinking to select the most promising ideas (Figure 2).

The terms Divergent and Convergent thinking were coined in the 1960s by J. Guilford, a psychologist known

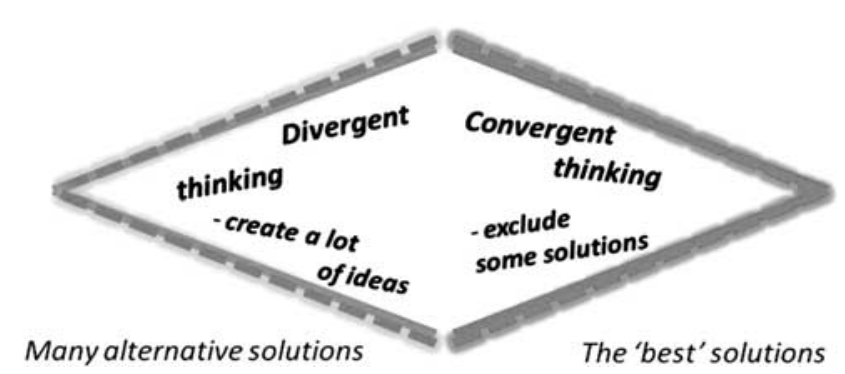

Figure 2 Divergent thinking and Convergent thinking. for his research on Creativity (Guilford, 1967). Divergent/ Convergent thinking phases have regularly been exploited in workshops and future conferences to promote creativity, innovation and strategy consensus (Vidal, 2006). Furthermore, they are expected as tactics of various methodologies for problem structuring and solving. Some of these issues are reported in Ferreira (2008) and Soares et al (2008).

Diversification and Intensification are components that appear in many Mh. The relations with Divergent/ Convergent thinking should be contemplated, since benefits may accumulate by analysing and combining procedures originating from different areas. Uncertainty is present in most situations faced by $\mathrm{Mh}$. A predisposition to tolerate and to deal with uncertainty may be gained from creativity, in particular from Divergent thinking and Convergent thinking. These issues will be addressed again in Section 5.

\subsection{Metaheuristics}

Metaheuristics (Mh) are general heuristic frameworks which can be applied to a wide variety of optimisation problems, with some modifications, and they are capable of escaping from local optima and performing a robust search of the solution spaces. They began to be introduced in the 1980s and 1990s; generic references include Blum et al (2008), Corne et al (1999), Dréo et al (2006), Glover and Kochenberger (2003), Gonzalez (2007), Hoos and Stultzle (2005), Reeves (1995), Talbi (2009), Zäpfel et al (2010). Examples of classical Mh include: Simulated Annealing (SA), Tabu Search (TS), Evolutionary Algorithms (EA), Memetic Algorithms (MA), Ant Colony Systems (AC), Scatter Search and Greedy Randomized Adaptive Search Procedure (GRASP), Hybrid Mh are also, nowadays a reference field for problem solving (Blum et al, 2008; Blum (Guest Editor), 2010). The topic of hybridising and exploiting different algorithms has also begun to appear regularly under the name Matheuristics (Boschetti et al, 2009; Maniezzo et al, 2010). Matheuristics are developed with the intension of benefiting from the interoperation of different methods: Mathematical Programming and $\mathrm{Mh}$. Hyper-heuristics also have recently attracted an increasing attention, due to the interest in developing general solvers which can handle classes of problems rather than solving just one problem. This should be the fundamental difference relative to Mh: most implementations of $\mathrm{Mh}$ just intend to solve a certain type of problems, whereas hyper-heuristics also search within a space of heuristics. They could be thought of as a (meta)heuristic which operates on lower level (meta-)heuristics (Burke et al, 2003).

$\mathrm{Mh}$ are the outcome of unlikely creative processes and merging ideas from different areas. In fact, many metaphors from nature and/or social behaviour have inspired these general heuristic methods. Their relevance, 
power and success are well-known, but there are some critical and practical questions without convenient responses: the choice of an Mh and, when a choice is made, how to improve the method, to elect adequate strategies or to tune its parameters. The quality of solutions and computational time are not the only criteria for analysing or selecting $\mathrm{Mh}$ and nor are they necessarily the most important. Certainly they are well-suited for questioning the efficiency and efficacy, but the effectiveness of a solution approach should also be evaluated from the perspective of practical problem solving. Other criteria should be taken into account and, quite often, they are intuitively used for real applications. However, the current weak theoretical results are of almost no practical use. In conclusion, there is no complete 'rational' guide to select or to implement an $\mathrm{Mh}$, or to hybridise $\mathrm{Mh}$, which may be seen as a weakness and not a positive sign (at least in the perspective of using other type of optimisation methods). It is our belief that a 'solution' or good practice will not emerge from the 'Mh background' alone and that significant benefits may be gained by the richer environment of Multimethodology.

The next sections expand these issues by mixing Soft methods and Creativity tools with 'hard' techniques, such as $\mathrm{Mh}$ in optimisation, what is not frequent. And this projected in a way more consentaneous with Multimethodology in parallel than in series, taking into account the work of Pollack (Pollack, 2009).

\section{Choosing Metaheuristics}

$\mathrm{Mh}$ are very powerful and flexible approximate techniques that can potentially be applied to any optimisation problem. Problem independence demands creativity. However, choosing and comparing or evaluating $\mathrm{Mh}$ is a difficult task. This is well-known but, in fact, there is a risk to credibility if no good answers are found. We expect to stimulate an interplay between different research fields, by showing some examples in Section 4. A few methods and tools will be briefly analysed that simultaneously organise properties and characteristics of a set of heuristics - those that are considered for a specific application. The intention is to handle quantitative and qualitative/soft information simultaneously. Information may arise from personal experience, publications and other sources.

Table 1 (a simple illustration) refers to solution methods, such as Local Search (LS), TS, SA, EA, MA and AS, and to a selection of possible characteristics or features. The characterisation/classification of those features is context dependent. For instance, 'Implementation simplicity' may well reflect the practitioner's skills and predilections for a particular Mh (the introduction of numbers in this table just aims to show the possibility of mixing quantitative and qualitative data - in this case $9 / 9$ is assigned to the simplest implementation of a group of applications). It is a simple tool which may be useful to arrange general characteristics and to facilitate team discussion relating to the potential of Mh. Notice that it is difficult, in general, to remain objective where the choice of an $\mathrm{Mh}$ is concerned.

Employing SWOT (Section 2) may also be recommended. As stated in Schulz (2006), the primary goal of SWOT will be to assist in choosing the Mh with the best possibility of implementing a divergent/convergent search strategy, a topic that will be discussed in Section 5 . Identifying and examining opportunities, threats, strengths and weaknesses (notions that should be adapted) are a fine groundwork for an agreement on choice and, finally, to help create a good implementation.

These tables may be completed for any $\mathrm{Mh}$ and can or cannot take into account, a specific application. Figure 3 (shortened) highlights the aim of applying Memetic Algorithms (Rodrigues and Ferreira, 2012) or other alternatives, such as Ant Colony Systems or the Frederickson's heuristic, to the Rural Postman Problem (RPP). The RPP will be defined in the next section as an illustrative application.

Uncertainties will be presented along with these tasks of choosing and comparing $\mathrm{Mh}$. The categorisation proposed by SCA, and mentioned earlier, may be of some help. Uncertainties pertaining to the working environment demand more accurate information. Examples of the manner in which such information may be sought are: surveys, research investigations and publications and $\mathrm{Mh}$ project implementations. Another type of uncertainty is related to choices on relevant agendas or decision fields: in this particular type, how decisions concerning areas such as modelling issues and practical exigencies, for instance, may affect the choice of solution methods are interesting.

Table 1 Comparing general characteristics for a specific application

\begin{tabular}{|c|c|c|c|c|c|c|}
\hline$M h+\ldots$ & $L S$ & $T S$ & $S A$ & $E A$ & $M A$ & $A S$ \\
\hline Neighbourhood search & yes & yes & yes & no & yes & no \\
\hline Capacity to escape local minima & - & & & & & \\
\hline Intensification/Diversification & & ++++ & + & ++ & +++ & + \\
\hline Implementation simplicity & $9 / 9$ & $7 / 9$ & $5 / 9$ & $6 / 9$ & $3 / 9$ & $4 / 9$ \\
\hline Case adaptation & (;) (;) ; & (;) (-) (-) & (;) :) & $\ldots$ & $\ldots$ & $\ldots$ \\
\hline
\end{tabular}




\begin{tabular}{|c|c|c|c|c|c|}
\hline$A C O$ & Opportunities & Threats & & \multirow{2}{*}{$\begin{array}{l}\text { Fred2-OPT } \\
\text { Strengths }\end{array}$} & \multirow[t]{2}{*}{ Oppor } \\
\hline Strengths & - & - & & & \\
\hline Weaknesses & - & - & & Weaknesses & \\
\hline & \multicolumn{2}{|c|}{ MA } & $\begin{array}{l}\quad \text { Opportunities } \\
\text { - easily applied } \\
\text { - intensification by LS } \\
\text {... }\end{array}$ & \multicolumn{2}{|c|}{$\begin{array}{l}\text { Threats } \\
\text { - long time in LS } \\
\text { (local search) } \\
\ldots\end{array}$} \\
\hline & \multicolumn{2}{|c|}{$\begin{array}{l}\quad \text { Strengths } \\
\text { - fittest survival convergence } \\
\text { - hybridisation } \\
\text { - modelling capabilities } \\
\text { - finds local optima } \\
\text {-- }\end{array}$} & $\begin{array}{l}\quad \text { Strategies } \mathrm{S}-\mathrm{O} \\
\text { - various local search } \\
\text { procedures } \\
\ldots\end{array}$ & \multicolumn{2}{|c|}{$\begin{array}{l}\text { Strategies S-T } \\
\text { ??? }\end{array}$} \\
\hline & \multicolumn{2}{|c|}{$\begin{array}{l}\quad \text { Weaknesses } \\
\text { - fittest survival convergence }\end{array}$} & $\begin{array}{l}\text { Strategies } \mathrm{W}-\mathrm{O} \\
\text { ??? }\end{array}$ & \multicolumn{2}{|c|}{$\begin{array}{l}\text { Strategies W-T } \\
\text { ??? }\end{array}$} \\
\hline
\end{tabular}

Figure 3 SWOT for the application of different heuristics to the RPP.

Table 2 Classification and comparison of different $\mathrm{Mh}$

\begin{tabular}{|c|c|c|c|c|c|c|}
\hline & Quality & Comp. effort & Simplicity & Adaptability & Robustness & Other? \\
\hline Mh1 & $\rightarrow$ & 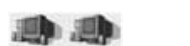 & S S & レレレレ & $\mathrm{rrrr}$ & - \\
\hline Mh2 & & 且 & s s s & $レ レ$ & $\mathrm{rrr}$ & - \\
\hline Mh3 & - & - & - & - & - & - \\
\hline Mh4 & $\rightarrow \vec{v}$ & 四 & $\mathrm{s}$ & レレ & $\mathrm{r} \mathrm{r}$ & - \\
\hline
\end{tabular}

It must be emphasised that these methods and tools are not supposed to present solutions or decisions, instead they encourage an expansion of the study to incorporate other aspects of the situation, transparency, participation with distinctive views and the organisation of non-quantitative knowledge and they may avoid the frequent rush into implementation.

\section{Comparing and evaluating Metaheuristics}

The Strategic Choice Approach (SCA) is a wellrecognised Soft OR methodology. As described in Section 2, it distinguishes four complementary working modes. Mh practice may benefit, in general, from the planning approach and from some of the concepts implicated in SCA. The Comparing mode and the Choosing mode are especially valuable to help cope with the complexity of comparing and evaluating $\mathrm{Mh}$. This will now be explained further.

Table 2 and Figure 4 illustrate tools that could be employed by individuals or groups to study, classify and evaluate $\mathrm{Mh}$.

As stated, other criteria besides the quality of solutions and the number of iterations or computational times should be examined and, quite often, they are intuitively used when dealing with practical optimisation problems. Accordingly, Table 2 may include qualitative and quantitative data - this is a very significant point which is very often disregarded. It is important to clarify what is meant

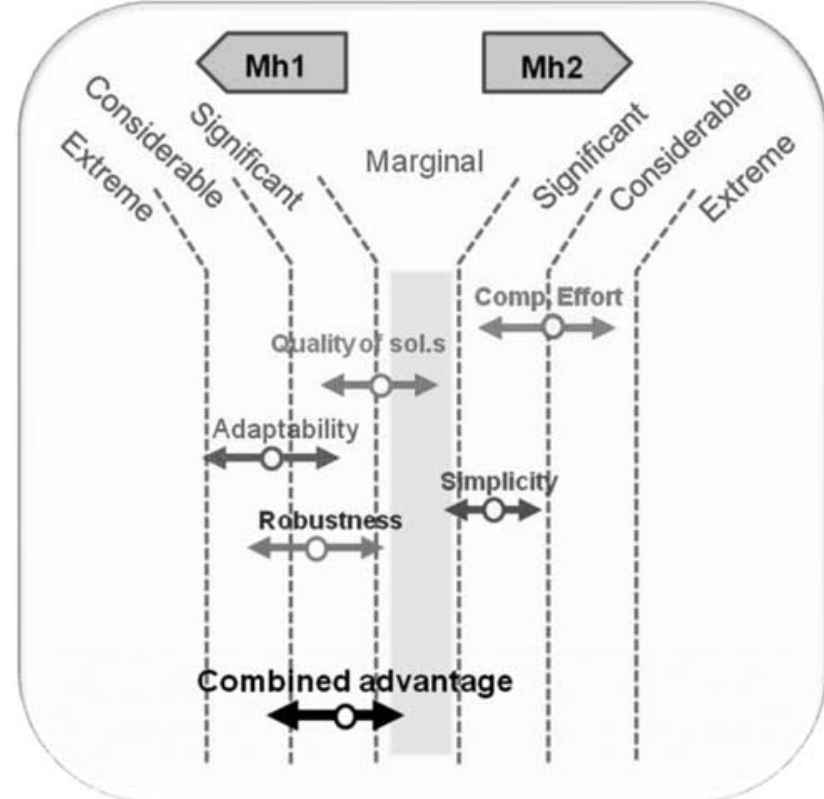

Figure 4 Advantage comparison grid for a selected pair (Mh1 and Mh2).

by each criterion. Eventually it may be more convenient to combine quality and computational effort into one criterion. As mentioned in Hooker (1995), 'algorithm experimentation should involve other measures in addition to solution quality and computational time and not just being a competitive test, that will only tell which heuristic 
algorithm is faster but not why'. The work (Smith-Miles and Lopes, 2012) provides a comprehensive survey of the experimental studies regarding the conditions under which an optimisation algorithm or search heuristic will succeed or fail. As mentioned, knowing these conditions and some hardness features of the problems is critical for understanding the strengths and weaknesses of different algorithms, and for automated algorithm selection. The relative importance of each criterion may eventually be defined/agreed on, in connection with a specific objective, application or client. Just by looking at Table 2, it may be concluded that Mh4 is dominated by Mh2, this one uses less computational effort, it is simpler, and it looks more robust. Three $\mathrm{Mh}$ are then left for more detailed analysis. If necessary, they may be compared/evaluated in pairs, using an advantage comparison grid or balance window, such as the one depicted in Figure 4. The grid allows for adjustments of positions and changes in ranges and a 'combined' row (below) indicates the mean position of all of the advantage assessments entered in the rows above. Appropriate formulas will aggregate the ranges. The software package STRAD (Strategic Adviser) may be of some help in the context of using SCA, especially if many $\mathrm{Mh}$ or criteria are under study.

The knowledge gained and the indications resulting from the previous use of another method, SWOT, may also be relevant for the purposes of this section. Observe that such a grid may not only be used for ongoing personal/team projects but also for organising and comparing results in published works. The 'working philosophy' of SCA, the ways of categorising and managing uncertainties, as previously mentioned, or to organise required activities (such as the Progress package), could also be taken into account.

Multiple criteria are involved here, but the aim is not to use any method to combine them into one, or to generate early consensus, or to transform all preferences into numbers; instead, $\mathrm{Mh}$ are compared pair-wise, relatively to all criteria, and the differences are ranked on a qualitative scale of relative advantage, ranging from 'marginal' to 'extreme'. This should become clear: the rationale is trying to look further for insights and to generate a richer way of handling the comparison and evaluation of $\mathrm{Mh}$, by allowing for a more effective intervention of the practitioner (or team). Decision-making supported by soft and hard data may become more comprehensible and transparent.

In order to demonstrate what has been suggested, a simple application based on current solution methods for the RPP is presented next. Another example, relating to the comparison of new methods for the Graph Partitioning Problem, is featured in (Mark et al, 2010a, b).

\subsection{Comparing methods to solve the RPP}

The RPP is more general than the Chinese Postman Problem, a well-known Arc Routing Problem, which consists of finding the shortest circuit that traverses each edge of a graph, at least once. These edges can be directed, undirected or both. In the case of the RPP, not all the edges have to be traversed by the circuit, only those in a specified subset of required edges. There are many applications of the RPP as referred in Corberan and Prins (2010) and Rodrigues and Ferreira (2010).

The following four methods, which are described in the respective references, are compared. It was possible to compare these effectively for each of the criteria.

- Fred2-OPT: solutions provided by the Frederickson algorithm in Frederickson (1979), followed by the 2-OPT improvement procedure of Hert et al (1999).

- INS2-OPT: solutions provided by the algorithm of Ghiani et al (2006), followed by the 2-OPT improvement procedure of Hertz et al (1999).

- $A C O$ : solutions provided by the ant colony optimisation algorithm ANTURPP in Laganà et al (2007).

- MA: solutions provided by the memetic algorithm MARP in Rodrigues and Ferreira (2012).

Four criteria (comparison areas) are introduced:

- Quality (solutions compared to the optimal solutions or tight bounds);

- Computational Efficiency (speed of computation);

- Simplicity (ease of implementation); and

- Robustness (performance across a large group of problems with different characteristics).

STRAD (version 2.3) was used. Figures 5-10 show some of the windows of that application. Figure 5 presents what is called an 'Assess Window'. It has different functions enabling, in particular, the entrance of a set of judgements/evaluations on the relative behaviour of each solution method (decision area) within each comparison area. Depending on how wide a rectangle has been made, one of the four words 'marginal, significant, considerable or extreme' will appear superimposed on it, reflecting the relative judgements made. For instance, considering the criterion Computational Efficiency, the most efficient method is $A C O$ (the widest

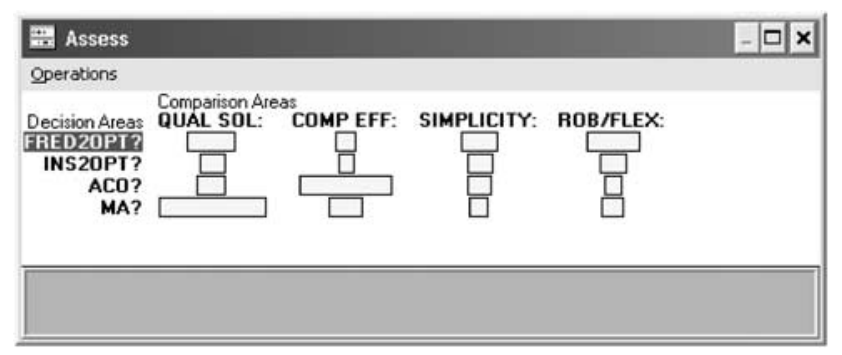

Figure 5 Assessment of the four solution methods. 
rectangle) followed by $M A$, Fred2-OPT and INS2$O P T$, according to the data of the above-mentioned publications.

INS2-OPT may be eliminated because it is dominated by Fred2-OPT. Therefore, three methods remain for advanced analysis: Fred2-OPT, $A C O$ and $M A$

Scenario 1 The evaluators attach the relative value weightings to the criteria (comparison areas) in Scenario 1, specified in Figure 6.

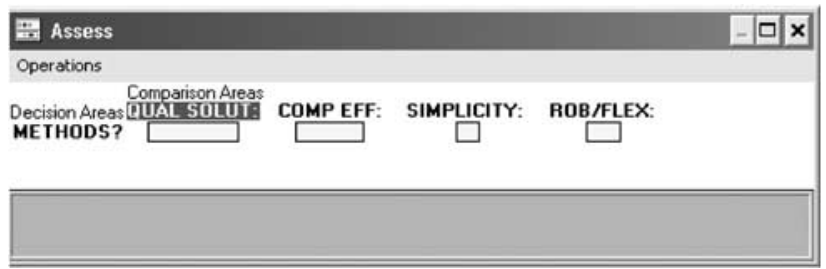

Figure 6 Adjusting the weightings (Scenario 1)
The four windows titled 'Options Assessment' in Figure 7 compare the methods for each criterion (units of measurement from 1 to 5 appear where specified). The part of the block covered by the yellow strip is proportional to the corresponding weight of the criterion.

The three solution methods are now compared two by two. The combined result is at the bottom of each of the 'Balance windows', in Figure 8.

This is the most essential of various other functions of the balance windows

The final window 'Combined Option Assessment' of Figure 9 gives preference to Fred2-OPT followed by $A C O$ and $M A$, this is obviously according to Scenario 1 .

Scenario 2 With regard to Scenario 2 different weightings (first window in Figure 10) are assigned to the criteria, for example, a large relevance to Simplicity, the ordering of preference should be Fred2-OPT, $M A$ and $A C O$ as depicted in Figure 10.

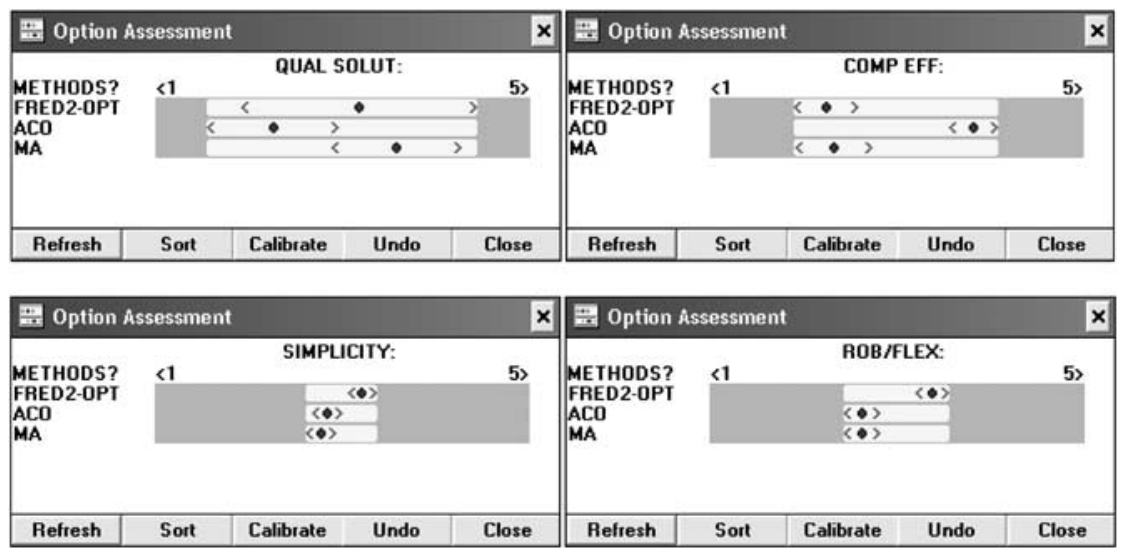

Figure 7 Position of the methods in relation to each criterion.

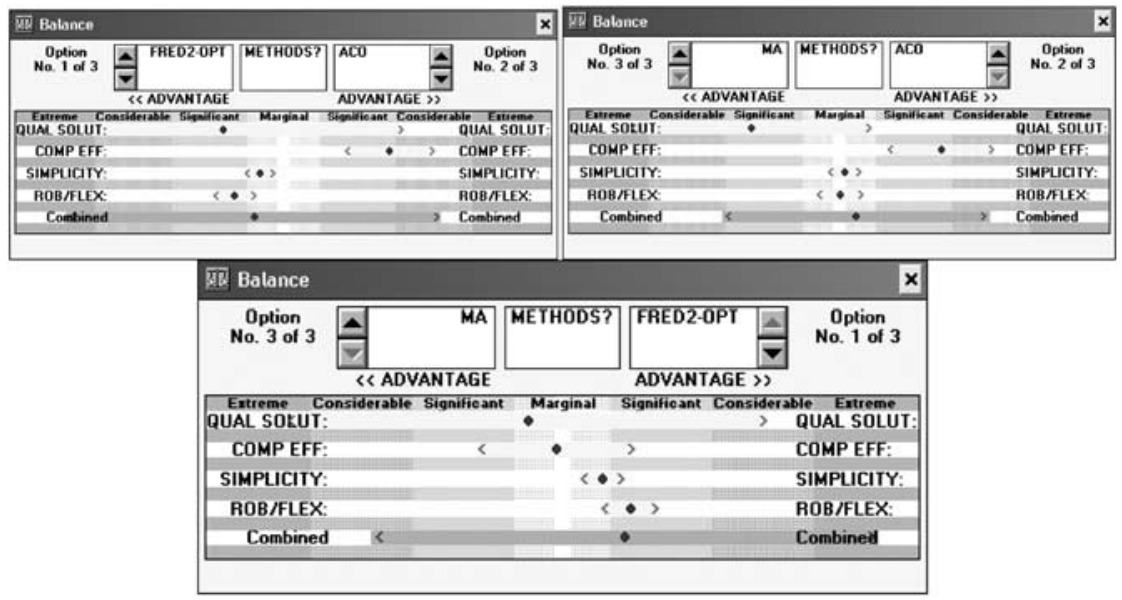

Figure 8 Combined evaluations of the methods (two by two). 


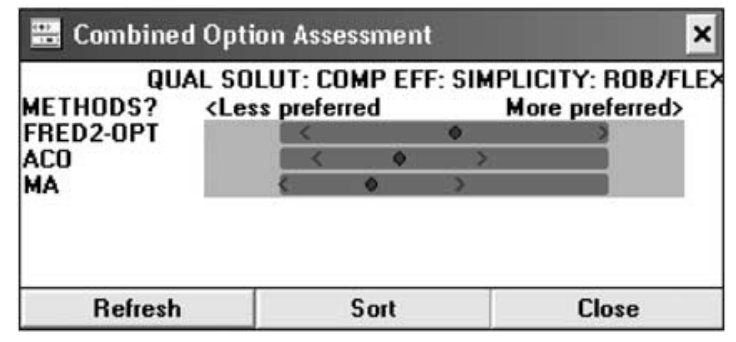

Figure 9 Final assessment (Scenario 1).

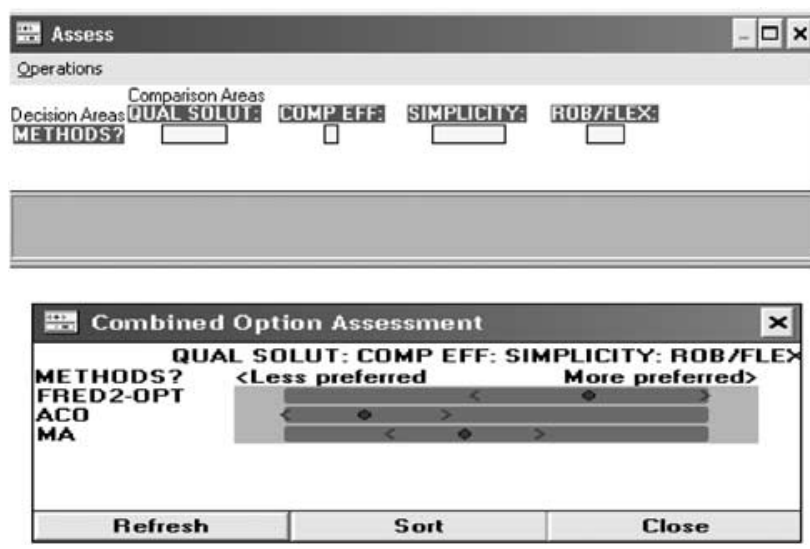

Figure 10 Final assessment (Scenario 2).

This application illustrated Multimethodology in practice. Appropriate functions of $S T R A D$ were used to compare a set of $\mathrm{Mh}$, according to different criteria, but the work could have been conducted without the software, by just following the SCA convenient steps.

\section{Implementing Metaheuristics}

Problem solving and implementing an $\mathrm{Mh}$ is a try-out and imprecise decision-making process - quantitative aspects are a good base; rational choices are interesting but, very often, they have their limitations; experience and intuition are frequently used, possibly without acknowledgement. The designer should be conscious that there are many aspects involved in the implementation of $\mathrm{Mh}$ that do not lend themselves to accurate planning. As mentioned earlier, a key support may be found outside the area. Divergent thinking/Convergent thinking, as part of CPS, may be helpful while planning, designing and tuning $\mathrm{Mh}$. Figure 2 is the source for a discussion on dealing with the solution of problems with Mh. Phases of Divergent and Convergent thinking should accompany the work - the researcher perceives that there is a dimension of creativity and adaptation in a project with $\mathrm{Mh}$. Brainstorming sessions involving the team elements at least are convenient for divergent phases. Figure 11 illustrates the continuum of the solution and the implementation process, noting that

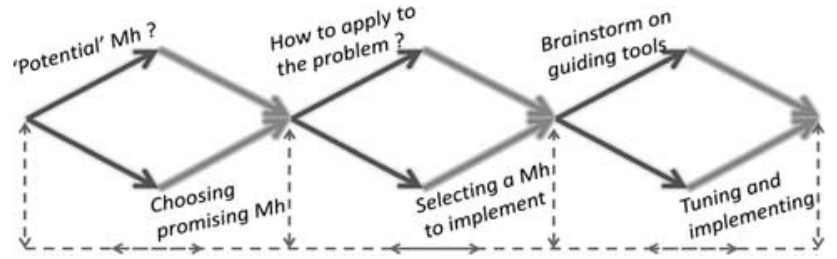

Figure 11 Solution and implementation process.

there are distinct steps or phases that should capture one's attention. This may be related to the work of Basadur et al (1994) which presents a methodology for problem solving as a four-stage process, emphasising the deferral of judgement, divergent thinking and convergent thinking at each stage.

Mh practice may undergo numerous revisions, between the most likely concentration on the set of principles of Mh, such as: Neighbourhoods - simple, extended,..., Search principles-local, systematic, random, populations of solutions ... Memories - adaptive, tabu lists, pheromone trails, ... the requirement of Parameter tuning and plausible steps towards Hybridisation. This iterative process is not essentially linked to problem-independent or problem-specific strategies, or whether the $\mathrm{Mh}$ are based on solution construction, modification or recombination. The aspects that should be taken into account in each of the phases have already been highlighted. Phases should be visited in turn (Figure 11); for instance, if one is following a convergent thinking phase in tuning the parameters, it may be convenient to undergo a new divergent phase on tuning, or even go back to a divergent thinking phase in 'guiding tools'. That is the advice from the feedback loops between all of the phases. Rushing up while choosing an Mh may not be convenient; if too much effort is used in tuning then the time has come to try another Mh and learn from the experience. The quest for the perfect configuration of parameter values will probably harm the 'essence' of Mh. Eventually a healthier way out will be the development of a dedicated heuristic, tailored to the specific problem or instance under study. A presentation and detailed application of the above-mentioned ideas is well reported in Schulz (2006), in the context of a search strategy for multi-modal continuous functions and for the optimisation of a ground water simulation model. We think that these procedures could also be employed in Hyper-heuristics projects.

The terms Divergent and Convergent thinking were coined in the 1960s by Guilford (1967). Diversification and Intensification concepts appeared later in connection with Tabu Search (TS) (see for instance Glover and Laguna, 1977). Diversification generally refers to exploration, the ability to visit many and different regions of the search space, whereas Intensification refers to the exploitation of the accumulated search experience to obtain high-quality solutions within those regions. Indeed, most $\mathrm{Mh}$ have 
several components for diversification and intensification, as mentioned in Lozano and García-Martínez (2010), a paper focusing on this subject. Finding a proper balance of diversification and intensification is a crucial issue in $\mathrm{Mh}$; achieving an effective interplay between divergent and convergent thinking is essential in CPS. Therefore, it could be interesting to reflect on a unifying view and the relations between these concepts, capacities and forms of thinking, that apparently originate from separate areas.

\section{Conclusions}

Multimethodology has developed significantly over the last decades. This paper aimed to show and to convey the idea that Multimethodology can also be useful in dealing with problem solving with Metaheuristics (Mh). Different soft and hard (partitioned) methodologies were combined, such as Soft OR methods, tools from Creativity and Mh. Adapted frameworks and applications were presented, which may play a crucial role while facilitating the communication and the discussion or by materialising a 'rational' to cope simultaneously with underlying hard and soft facets. The SWOT Analysis helped choose a convenient method from a collection of $\mathrm{Mh}$. Selecting $\mathrm{Mh}$ also requires the know-how, the preferences and the intuition of the user, rather than the application of well-established rules. Tools from Strategic Choice Approach to deal with the comparison or evaluation of $\mathrm{Mh}$ (beyond the frequent race for high-quality solutions) can be helpful. A dynamic framework based on Creativity methods, such as Divergent and Convergent thinking, can be used to guide, adjust and assist their implementation. As mentioned, the approach followed in the paper is more close to a parallel way of combining distinct methods, hard and soft, than a serial one.

It is apparent that much more can be done in this line of research, eventually extending it to Hyper-heuristics (the choice and evaluation of heuristics is essential) and that the overall problematic linked with $\mathrm{Mh}$ practice, from choice to design and implementation, may overtake the disciplinary boundary of $\mathrm{Mh}$ and encourage the use of Multimethodology.

\section{References}

Ackoff R (1978). The Art of Problem Solving. Wiley: New York.

Ackoff R (1987). OR: A post mortem. Operations Research 35(3): 471-474.

Ackoff R and Vergara E (1981). Creativity in problem solving and planning: A review. European Journal of Operational Research 7(1): 1-13.

Basadur M, Ellspermann S and Evans G (1994). A new methodology for formulating ill-structured problems. Omega 22(6): 627-645.

Bell G, Warwick J and Kennedy M (2009). From theory to practice and back: Some experiences with an emerging multimethodology. OR Insight 22(2): 65-87.
Blum C (Guest Editor) (2010). Hybrid metaheuristics. Computers \& Operations Research 37(3): 429-610

Blum C, Aguilera B, Roli M and Sampels M (eds). (2008). Hybrid Metaheuristics - An Emerging Approach to Optimization. Studies in Computational Intelligence. Vol. 114. Springer: New York.

Boschetti M, Maniezzo V, Roffilli M and Röhler A (2009). Matheuristics: Optimization, simulation and control. In: Proceedings of HM 2009. LNCS 5818, Springer-Verlag: New York, pp 171-177.

Burke E, Hart E, Kendall G, Newall J, Ross P and Schulenburg S (2003). Hyper-heuristics: An emerging direction in modern search technology. In: Glover F and Kochenberger G (eds). Handbook of Metaheuristics. Kluwer: Dordrecht, MA, pp 457-474.

Corberan A and Prins C (2010). Recent results on arc routing problems: An annotated bibliography. Networks 56(1): 50-69.

Corne D, et al (eds). (1999). New Ideas in Optimization. McGrawHill: New York.

De Bono E (1992). Serious creativity: Using the Power of Lateral Thinking to Create New Ideas. HarperCollins: New York.

Dréo J, Pétrowski A, Siarry P and Taillard E (2006). Metaheuristics for Hard Optimization. Springer: New York.

Driscoll D, Appiah-Yeboah A, Salib P and Rupert D (2007). Merging qualitative and quantitative data in mixed methods research: How to and why not. Ecological and Environmental Anthropology 3(1): 19-28.

Eden C, Ackermann F, Bryson J, Richardson GD, Andersen D and Finn C (2009). Integrating modes of policy analysis and strategic management practice: Requisite elements and dilemmas. Journal of the Operational Research Society 60(1): 2-13.

Ferreira JS (2008). Creativity in Optimization-Some Ideas. IV SELASI-European Latin American Workshop on Engineering Systems, 1-5 December, Havana, Cuba.

Frederickson G (1979). Approximation algorithms for some postman problems. Journal of the Association for Computing Machinery 26(3): 538-554.

Friend J (2001). The strategic choice approach. In: Rosenhead J and Mingers $\mathbf{J}$ (eds). Rational Analysis for a Problematic World Revisited. John Wiley: New York, pp 115-150.

Friend J and Hickling A (2005). Planning Under Pressure: The Strategic Choice Approach. Elsevier: Amsterdam.

Ghiani G, Laganà D and Musmanno R (2006). A constructive heuristic for the undirected rural postman problem. Computers \& Operations Research 33(12): 3450-3457.

Glass R (2006). Software Creativity 2.0. developer.* Books: Atlanta, GA.

Glover F and Kochenberger G (eds). (2003). Handbook of Metaheuristics. Kluwer: Amsterdam.

Glover F and Laguna M (1977). Tabu Search. Kluwer Academic Press: Dordrecht, MA.

Gonzalez T (eds). (2007). Handbook of Approximation Algorithms and Metaheuristics. Chapman: London.

Gordon W (1961). Synetics: The Development of Creative Capacity. Harper \& Row: New York.

Guilford J (1967). The Nature of Human Intelligence. McGraw-Hill: New York.

Herrmann N (1996). The Whole Brain Business Book. McGrawHill: New York.

Hertz A, Laporte G and Hugo P (1999). Improvement procedures for the undirected rural postman problem. INFORMS Journal on Computing 11(1): 53-62.

Hooker J (1995). Testing heuristics: We have it all wrong. Journal of Heuristics 1(1): 33-42.

Hoos H and Stultzle T (2005). Stochastic Local Search: Foundations and Applications. Elsevier: Amsterdam. 
Howick S and Ackermann F (2011). Mixing OR methods in practice: Past, present and future directions. European Journal of Operational Research 215(3): 503-511.

Johnson R and Onwuegbuzie A (2004). Mixed methods research: A research paradigm whose time has come. Educational Researcher 33(7): 14-26.

Joldersma C and Roelofs E (2004). The impact of soft OR-methods on problem structuring. European Journal of Operational Research 152(3): 696-708.

Keys P (2006). On becoming expert in the use of problem structuring methods. Journal of the Operational Research Society 57(7): 822-829.

Kotiadis K and Mingers J (2006). Combining PSMs with hard OR methods: The philosophical and practical challenges. Journal of the Operational Research Society 57(7): 856-867.

Laganà D, Laporte $\mathrm{G}$, Mari F, Musmanno R and Pisacane O (2007). An ant colony optimization metaheuristic for the undirected rural postman problem. Les Cahiers du GERAD, G-2007-106.

Lozano M and García-Martínez C (2010). Hybrid metaheuristics with evolutionary algorithms specializing in intensification and diversification: Overview and progress report. Computers \& Operations Research 37(3): 481-497.

Mabin V, Davies J and Kim S (2009). Rethinking tradeoffs and OR/MS methodology. Journal of the Operational Research Society 60(10): 1384-1395.

Maniezzo V, Stützle T and Voß S (eds). (2010). Matheuristics Hybridizing Metaheuristics and Mathematical Programming. Series: Annals of Information Systems, Vol. 10. Springer: New York.

Marakas G and Elam J (1997). Creativity enhancement in problem solving: Through software or process? Management Science 43(8): 1136-1146.

Mark M, Pedroso J and Ferreira JS (2010a). New methods to solve the graph partitioning problem. EURO XXIV, July 11-14, 2010, Lisboa, Portugal.

Mark M, Pedroso J and Ferreira JS (2010b). The graph partitioning problem - Comparing solution methods. FEUP/INESC TEC.

Mingers J (2000). Variety is the spice of life - Combining soft and hard OR/MS methods. International Transactions in Operations Research 7(6): 673-691.

Mingers J (2001). Multimethodology-Mixing and matching methods. In: Rosenhead $\mathbf{J}$ and Mingers $\mathbf{J}$ (eds). Rational Analysis for a Problematic World Revisited. John Wiley and Sons: Chichester, pp 289-309.

Mingers J and Brocklesby J (1997). Multimethodology: Towards a framework for mixing methodologies. OMEGA 25(5): 489-509.

Mingers J and Gill A (eds). (1997). Multimethodology: Theory and Practice of Combining Management Science Methodologies. Wiley: Chichester.

Mingers J, Liu W and Meng W (2009). Using SSM to structure the identification of inputs and outputs in DEA. Journal of the Operational Research Society 60(2): 168-179.

Munro I and Mingers J (2002). The use of multimethodology in practice-Results of a survey of practitioners. Journal of the Operational Research Society 53(4): 369-378.

Ormerod R (2001). Mixing methods in practice. In: Rosenhead J and Mingers $\mathbf{J}$ (eds). Rational Analysis for a Problematic World Revisited: Problem Structuring Methods for Complexity, Uncertainty and Conflict. Wiley: Chichester, pp 289-310.

Osborn A (1953). Applied Imagination. Charles Scribner's \& Sons: New York.

Panagiotou G (2003). Bringing SWOT into focus. Business Strategy Review 14(2): 8-10.
Papamichail K, Alves G, French S, Yang J and Snowdon R (2007). Facilitation practices in decision workshops. Journal of the Operational Research Society 58(5): 614-632.

Parnes S (1997). Optimize: The Magic of Your Mind. Bearly Limited: Buffalo, NY.

Paucar-Caceres A (2009). Mapping the changes in management science: A review of 'soft' OR/MS articles published in Omega (1973-2008). Omega 38(1-2): 46-56.

Pidd M (1996). Tools for Thinking. Wiley: New York.

Pollack J (2009). Multimethodology in series and parallel: Strategic planning using hard and soft OR. Journal of the Operational Research Society 60(2): 156-167.

Reeves C (eds). (1995). Modern Heuristic Techniques for Combinatorial Problems. McGraw-Hill: New York.

Rodrigues A and Ferreira JS (2010). Rural postman and related arc routing problems. EURO XXIV, 11-14 July, Lisboa, Portugal.

Rodrigues A and Ferreira JS (2012). Cutting path as a rural postman problem: Solutions by memetic algorithms. International Journal of Combinatorial Optimization Problems and Informatics 3(1): 22-37.

Rosenhead J and Mingers J (eds). (2001). Rational Analysis for a problematic World Revisited. John Wiley: New York.

Rubinstein M (1986). Tools for Thinking and Problem Solving. Prentice-Hall: Englewood Cliffs, NJ.

Runco M (2007). Creativity. Elsevier: Amsterdam.

Schulz P (2006). Creative design in optimization-Metaheuristics design to multi-modal continuous functions. Technical University of Denmark, IMM-M.Sc, 2006-2029.

Smith-Miles K and Lopes L (2012). Measuring instance difficulty for combinatorial optimization problems. Computers \& Operations Research 39(5): 875-889.

Soares G, Rodrigues A and Ferreira JS (2008). Facilitating to deal with combinatorial optimisation problems: Report of a workshop (in Portuguese). INESC TEC.

Sodhi M and Tang C (2008). The OR/MS ecosystem: Strengths, weaknesses, opportunities, and threats. Operations Research 56(2): 267-277.

Talbi E (2009). Metaheuristics: From Design to Implementation. Wiley: New York.

Teddlie C and Tashakkori A (2009). Foundations of Mixed Methods Research: Integrating Quantitative and Qualitative Approaches in the Social and Behavioral Sciences. Sage Publications: London.

Vidal V (Guest Editor). (2004). European Journal of Operational Research 152(3): 529-806.

Vidal V (2004). Applications of Soft O.R. Methods. Creativity for operational researchers. Investigação Operacional 25(1): 1-24.

Vidal V (2006). Creative and Participative Problem SolvingThe Art and the Science, http://www2.imm.dtu.dk/ vvv/CPPS/ index.htm.

Weihrich H (1982). The TOWS matrix - A tool for situational analysis. Long Range Planning 15(2): 54-66.

White L (2009). Understanding problem structuring methods interventions. European Journal of Operational Research 199(3): 823-833.

Winterfeldt D and Fasolo B (2009). Structuring decision problems: A case study and reflections for practitioners. European Journal of Operational Research 199(3): 857-866.

Zäpfel G, Braume R and Bögl M (2010). Metaheuristic Search Concepts. Springer: New York.

Received October 2010; accepted June 2012 after two revisions 